\title{
Automatic diabetic retinopathy detection and classification system
}

\begin{abstract}
Diabetic Retinopathy (DR) is an eye disease due to diabetes, which is the most ordinary cause of blindness among adults of working age in Malaysia. To date, DR is still screened manually by ophthalmologist using fundus images due to insufficiently reliable existing automated DR detection systems. However, the manual screening process is the weakest link as it is a complicated and time-consuming process. Hence, this paper proposed an algorithm that consists of DR detection method with the aim to improve the accuracy of the existing systems. The methods used to detect DR features, namely exudates, hemorrhages and blood vessels can be categorized into several stages which are image pre-processing, vessel and hemorrhages detection, optic disc removal and exudate detection. However, the detection for blood vessel and hemorrhages was performed simultaneously due to similar intensity characteristics. The proposed algorithm was trained and tested using 49 and 89 fundus images, respectively. The images used in training were obtained from Hospital Serdang, Malaysia while images used in the testing were obtained from DIARETDB1 database. All of the images were categorized into four DR stages, namely mild Non-Proliferative Diabetic Retinopathy (NPDR), moderate NPDR, severe NPDR and Proliferative Diabetic Retinopathy (PDR). The images were captured under various illumination conditions. In the testing, the result shows that the percentage of detection for blood vessel and hemorrhages, and exudates are $98 \%$ and $100 \%$, respectively.
\end{abstract}

Keyword: Diabetic retinopathy (DR); Exudates; Fundus images; Hemorrhages; Blood vessels; Microaneurysms; Detection; Pre-processing 